\title{
Article \\ The New Gear Finishing Method Research for Highly Loaded Gears
}

\author{
Wieslaw Budzisz * and Adam Marciniec (D)
}

Citation: Budzisz, W.; Marciniec, A. The New Gear Finishing Method Research for Highly Loaded Gears. Aerospace 2022, 9, 131. https:// doi.org/10.3390/aerospace9030131

Academic Editor: Stelios

K. Georgantzinos

Received: 3 February 2022

Accepted: 23 February 2022

Published: 3 March 2022

Publisher's Note: MDPI stays neutral with regard to jurisdictional claims in published maps and institutional affiliations.

Copyright: (C) 2022 by the authors. Licensee MDPI, Basel, Switzerland. This article is an open access article distributed under the terms and conditions of the Creative Commons Attribution (CC BY) license (https:// creativecommons.org/licenses/by/ $4.0 /)$.
Department of Mechanical Engineering, Faculty of Mechanical Engineering and Aeronautics, Rzeszow University of Technology, Al. Powstancow Warszawy 8, 35-959 Rzeszow, Poland; amarc@prz.edu.pl

* Correspondence: d456@stud.prz.edu.pl

\begin{abstract}
Achieving roughness parameters on tooth flanks for highly loaded gears and creating their non-directional surface texture are key to obtaining the required gearbox lifetime, durability, and performance. Many finishing techniques are well-known and described in technical literature, including gear teeth final grinding, shaving, honing, and superfinishing to create the proper surface condition. Unfortunately, there is still a lack of a simple finishing method which can be applied to highly loaded gears in the manufacturing process in order to optimize cost, time, and waste. This work presents the effect of a non-chemical, abrasive, highly effective process to remove quite a large amount of material from gear teeth flanks. The researched technology is called Stream Finishing (SF). The surface condition was inspected to observe its properties before and after the proper SF process parameters definition. It is useful for transmissions groups to define the proper manufacturing process application for obtaining the required gearbox lifetime within optimized parts cost. For each application the process parameters definition shall be determined individually and tested.
\end{abstract}

Keywords: gear; roughness; superfinishing; stream finishing; surface analysis; topography; involute; teeth flank

\section{Introduction}

Throughout human history, gears have always played an extremely important role when it comes to transmitting power or movement at the proper speed. These are very important constructions, developed over years, which are subject to high requirements depending on the type of gear and their uses. Gears are used to transmit power from the engine or motor to the working machine and provide the correct speed necessary for proper operating conditions. Depending on the type of application, gears can have straight teeth, single-flanged helical teeth, or double helical teeth, whereby the use of such a solution reduces axial forces. Gears may be designed as coaxial wheels when it is necessary to transmit the torque without changing the direction of the speed. However, when it is necessary, bevel gears with perpendicular axes can be used as well. Depending on the type of application, gears can be exposed to either low torques, as in the automotive and food industries, or strong forces found in heavy, marine, and aviation industries. In the agricultural industry, gears work in specific conditions with a lot of contaminations, dirty water, and dustiness by sand or soil [1-3].

Depending on their applications, the gears used in aircraft construction have a very narrow tolerance range and are sensitive to misalignment [4,5]. Unexpected in-flight events are also considered a risk to proper gear design in aviation. To ensure their required service life, gears with high rotational speeds require flawless surfaces on their tooth flanks, which can be achieved using special state-of-the-art superfinishing processes. Lubricated oils work under pressure and have a much thinner consistency than the oils used in the automotive industry. In automotive application, oil film breakage is less risky and less likely than in aircraft transmissions [6,7]. Another type of gearbox is the planetary system, where the sun gear drives the planet gear. Here, the sun gear is the input gear, and the hollow 
gear is the power receiving part. Planetary gears transmit high power while obtaining significant gear ratios. The planetary gear is still quite simple in design (a lot of design books help to create the proper constructions supported by 3D software) but is technologically complicated enough for high quality product applications (Figure 1). In the aviation industry, gear design also becomes very complicated due to operating conditions (high speeds, transmitted power, unexpected events, vibrations, specific lubrication method and fluid, sliding velocities issues, micropitting, and scuffing in high sliding velocity areas) and misalignments in operation conditions $[8,9]$. This leads to proper contact ratio and backlash determination, understanding of mesh stiffness variation, and influence of manufacturing errors [10,11]. In addition, gears in aviation work with much higher bending stress level, which requires the detailed finishing of the gear's top lands and of the break edges from the gears' faces. For scuffing and pitting avoidance, the proper surface topography achievement—surface lay, waviness along gear teeth involute, roughness-is required [12].

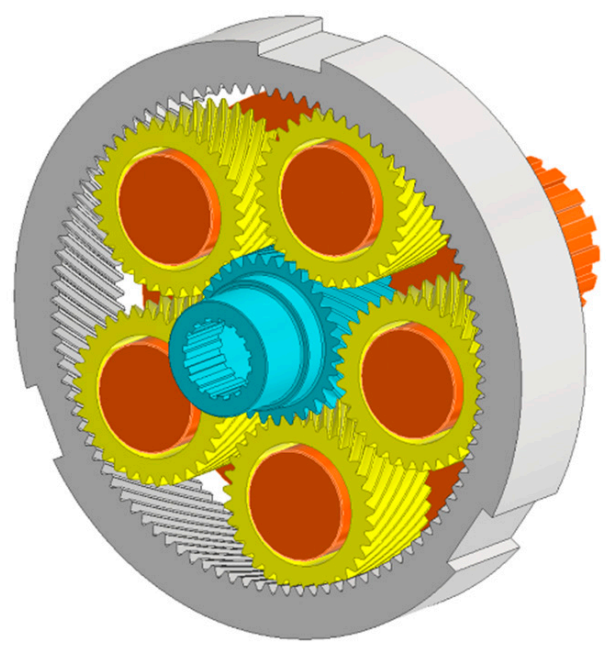

Figure 1. KISSsoft virtual 3D model of typical planetary gearbox.

\section{Materials and Methods}

The workpieces in the researched subject were gears of 5 inch pitch diameter, made from carburizing steel with high case hot hardness, high surface hardness, abrasion resistance and excellent resistance for tempering. The initial surface prior to the Stream Finishing (SF) process was machined within Ra $0.27 \mu \mathrm{m}$ average. There were three workpieces and each was subjected to a different SF process parameter at the optimum time to make the manufacturing process optimized when compared to other processes available on the gear manufacturing market [13]. Finally, one of the three workpieces was defined as the optimized surface, representative of optimized SF process parameters. Specific future applications should include the statistical analysis of the parts to observe the material removal rate variation from the involute (part-to-part variation). However, for workpieces the involute tooth-to-tooth shape variations are within a small range compared to the involute tolerance in highly loaded gears in aviation. It means that the SF process is quite repeatable.

There are many different technological processes for finishing the surfaces of gear teeth. The first well-known process is the final teeth grinding operation using a contouring or circumferential method. The grinding wheels can be CBN or ceramic discs. For gear surfaces subject to high power and speed, a honing process is used to reduce the machining marks after tooth grinding and to create the required topography of the tooth flanks and achieve the correct roughness Ra. It has a very beneficial effect on gear performance and gearbox lifetime when honing is applied. For very heavily loaded gears, honing is insufficient because it still delivers too high Ra values. Therefore, more precise machining 
is required, which provides about half of the surface roughness that honing does. One of the methods available on the market is superfinishing. This process generates a good surface that is suitable for highly loaded gears with isotropic topography. However, it is sometimes difficult to implement and too complex for specific components, and hard to achieve the extremely high product quality within specification requirements. On the other hand, this method provides a very good surface condition which ensures high gearbox performance [14,15].

One more method is being researching now and it is described in this paper. The proposed solution will help to finish the parts in specific applications within a very tight quality requirement. The research description of the new finishing method is presented in this paper, as well as the gear teeth flank conditions resulting from the process. It is called Stream Finishing (SF). The main benefit of the proposed solution is optimized Ra. It is two times better for Ra than grinding, about $50 \%$ better than honing, and comparable to superfinishing. However, it is environmentally friendly because the fluid works in a closed loop, thus optimizing waste. The workpieces for this process were middle-sized gears. The gears were installed on the machine spindle in different positions to determine the proper parameters which help to effectively remove the material from the gear teeth flanks. The process parameters were modified by proper adjustments after initial tests which had produced unacceptable results. The research includes and considers all engineering requirements of the workpiece: required $\mathrm{Ra}$, isotropy of the surface, lack of residual grinding process lines, surface irregularities (valleys) to accommodate lubrification; and lack of unacceptable irregularities on the teeth flanks for highly quality specifications, in order to determine the proper SF process parameters. There are several important benefits of the SF method. The main advantage of this process is its simplicity. Dedicated machines can be precisely programmed for a very controlled spindle movement, and it can be integrated with a production line because it works in a very stable way, without vibrations. The workpiece is mounted on a spindle that allows a wide variety of clamping concepts, such as collets, HSK interfaces, or zero-point clamping systems. The spindle starts rotating and immersing the workpiece in a rotating drum filled with an abrasive medium. A rigid spindle which performs a rotating motion, a drum filled with a constant level of the medium and the angular position of the spindle in relation to the drum axis are all very simple parameters to control, which makes the process stable, repeatable, easily predictable, and easy to change. In addition, the lack of chemical usage means that there are no problems with their mixing, handling and logistics. The liquid filling the drum circulates in a closed circuit. The fluid is a simple mixture of demineralized (demi) water and a compound which contains surfactants and a corrosion inhibitor. A centrifugal cleaning system separates the solid waste (metal abrasion and worn media) from the process water. This allows for reuse and circulation of the water, which makes the whole system environmentally friendly. Figure 2 presents the SF process equipment, with description of the main parts and machine modules. Figure 3 presents the centrifugal fluid cleaning machine for the SF process, an additional part of equipment used for environmental benefit and logistical improvement [16]. 


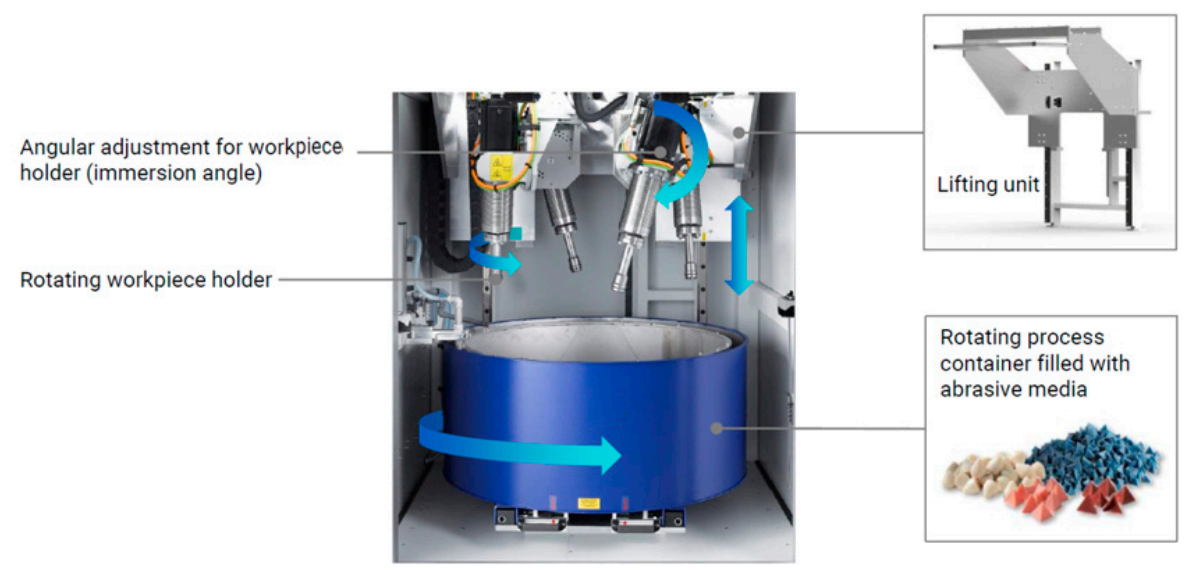

Figure 2. Stream Finishing process equipment [17].

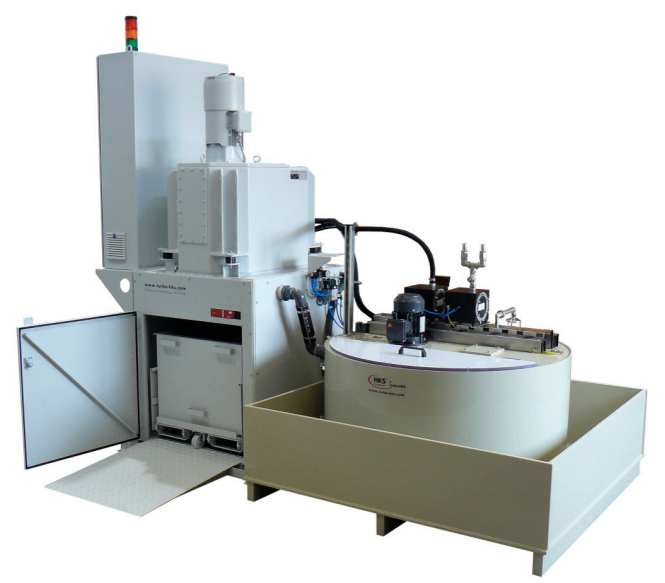

Figure 3. The centrifugal cleaning system which separates process water from solid waste [17].

The workpiece is in the drum and mounted on the spindle by a simple assembly system. The spindle can be adjusted by a specific angle to the rotation axis of the drum. Adjusting the appropriate spindle settings enables a constant flow of the medium through the workpiece geometry, which is very beneficial for the repeatability of the process. The main aspect is the fact that to machine the workpiece requires immersing this in the drum of the machine. The workpiece is in a fixed position in the machine's coordinate system. Compared to other applications on the market where the workpiece can move freely in drum, the SF system thus guarantees the repeatability of the process and the very controlled movement of the workpiece when mounted on the spindle (as presented in Figures 4 and 5).

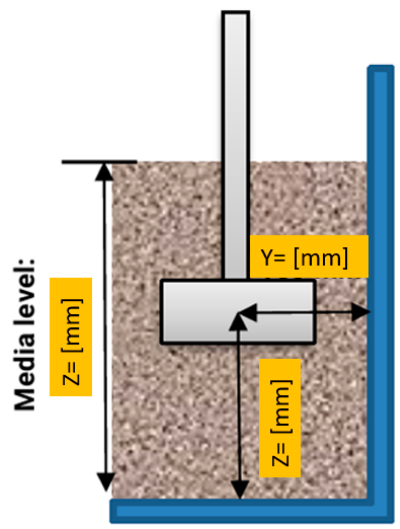

Figure 4. Workpiece location in the drum [17]. 

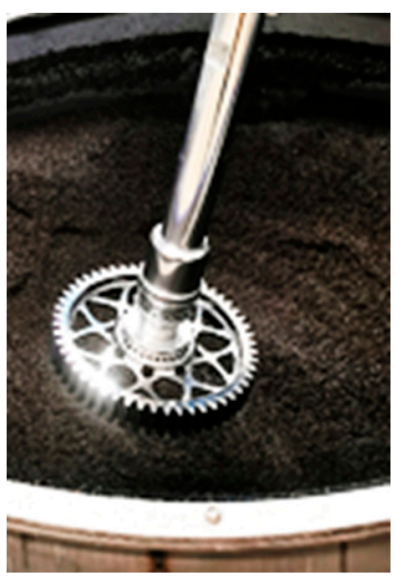

Figure 5. Spur gear as the workpiece mounted on Stream Finishing machine spindle [17].

The abrasive medium used in the SF process is aluminum oxide in the form of small particles which remove the material from the surface by friction between the medium and the workpiece. Depending on the gear module, it is possible to use different medium sizes. The great advantage of this process is the fact that small particles of abrasive medium can reach into the gear root diameter areas, which is problematic for the most common industrial gear finishing processes. It ensures an appropriate treatment and visual shape, which is very beneficial in terms of bending stresses. Furthermore, the tooth edges (at the tip and the gear faces) tend to provide even, repetitive curves, which is also very beneficial. (notching effect). The SF process provides proper topography and roughness. Figure 6 presents the medium piece shapes and Figure 7 presents the medium size versus high gear teeth.

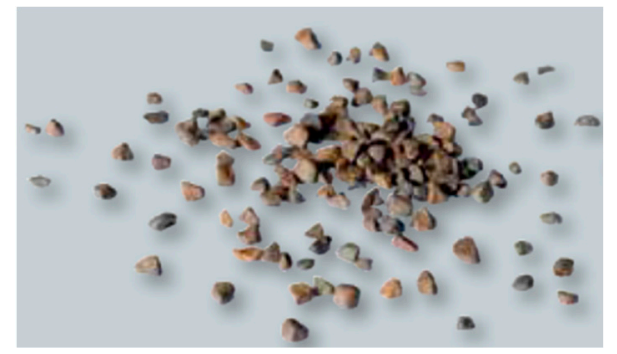

Figure 6. Medium in sizes 1.7-2.4 mm marked as KXMA 16 [17].

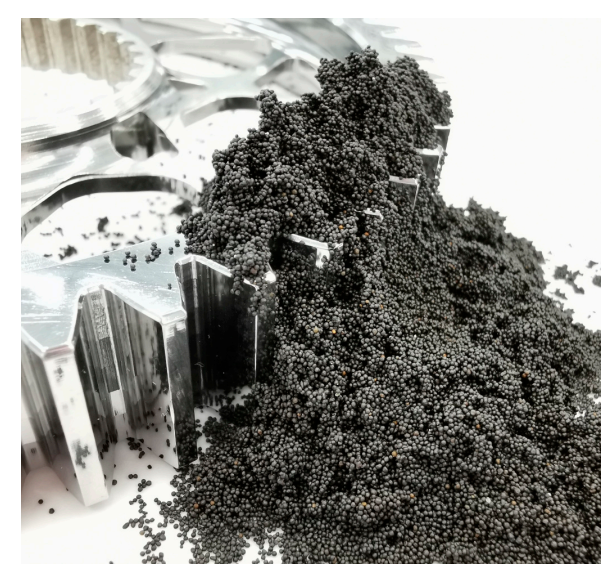

Figure 7. Medium size versus gear teeth module [17]. 
One of the possible ways to examine the surface finish condition after the SF method has been applied in the research is the contact method. The measuring tip is moved at a constant speed over the surface of the workpiece. Using the contact measurement method, a sensor of the roughness measuring device touches the surface point by point [18]. The screen of the machine easily detects roughness parameters such as $\mathrm{Ra}, \mathrm{Rp}, \mathrm{Rv}, \mathrm{Rz}$. In addition, involute waviness and primary profile parameters could be checked and compared in the research before and after the proper SF process parameters determination. A stereoscopic microscope was applied to observe any irregularities of the surface, residual grinding lines, any surface defects generated in the SF process, or any residual irregularities from the final teeth grinding. By using a specific light angle, it is very easy to observe the surface to verify the initial isotropy level [19]. A confocal method was also applied to measure 3D surface parameters, creating a very good visualization of the surface and to measure the level of isotropy by isotropic chart preparation. The visualization in different color, scales, and views is helpful in order to observe the waviness pattern [20]. A scanning electron microscope (SEM) is very useful to understand the media flow which SF obtained. Non-directional flow is desirable. SEM can also check for any residual media pieces stocked on the teeth flanks which could generate three-body abrasive wear [21,22]. An atomic force microscope (AFM) was used to observe oil-lubricated valleys on the measured surface [23]. These devices, which were very useful in the present research, are widely described in technical literature. Therefore, this paper does not include extensive details about them [24].

\section{Results}

The durability and reliability of the gear depend on its tribological properties to resist the gear teeth flanks against pitting and scuffing $[25,26]$. Additionally, the teeth are subjected to extremely high bending stresses. Gear tooth flanks of highly loaded gears require proper gear teeth topography. Ra parameter is important, but also the isotropy of the surface, the shape of the surface irregularities, the values of the peaks and valleys of unevenness defined by additional roughness parameters and the shape of the involute in relation to waviness and primary profile. The presented parameters affect friction and wear of the two surfaces, impact lubrication, mechanical tightness and transmission assembly [27]. The optimized Stream Finishing (SF) process for the workpieces requires the basic selection of the proper medium type, the spindle angle adjustment, the level of medium in the container, the workpiece distance from the container walls, as well as the selection of the process method (wet finishing or dry finishing) in order to find the minimum involute waviness and roughness on the gear tooth flanks. Table 1 presents gear teeth parameters before and after optimized SF process determination related to ground surface. The measuring system device was the standard roughness inspection machine (Mahr) with $5 \mu \mathrm{m}$ tip radius of a stylus. It measured areas near to sliding velocities in $25 \%$ and $75 \%$ face width sections. Non-optimized process parameters (values in red) had given waviness and primary profile parameters outside the incoming range. This happened when too-small aggressive media flow was used on the teeth flanks. The amount of the material from the surface was not removed uniformly and effectively (it just followed the high peaks of waviness generated in grinding process). This surface condition is not adequate to avoid gear damage. The optimized process established the values in the proper range (values in green). The visibility of unidirectional grinding scratches is unfavorable in gears. The waviness of the involute would influence many factors. One of them is the uneven load distribution along the involute from a steady, computational state, causing local concentrations of contact stresses, affecting risks of fatigue, such as pitting, micropitting, and abrasive wear or local wear, leading to the destruction of the gear. Another example is the increase of bending stress at tooth fillet radius area by the tooth bending forces applied to a local peak of the involute irregularities. Another aspect is the undesirable vibrations of the gearbox due to transmission errors and the risk of breaking the oil film, causing scuffing and local abrasive wear leading to destruction of the transmission [28]. 
Table 1. Surface texture parameters after Stream Finishing vs ground surface. Values are in $\mu \mathrm{m}$.

\begin{tabular}{|c|c|c|c|}
\hline Parameter & Ground Surface & Optimized SF & Non-Optimized SF \\
\hline $\mathrm{Ra}$-arithmetic mean roughness value & 0.27 & 0.09 & 0.09 \\
\hline $\mathrm{Rq}$-root mean squared roughness value & 0.41 & 0.12 & 0.11 \\
\hline $\mathrm{Rz}$-maximum height of roughness profile & 2.60 & 0.59 & 0.60 \\
\hline Rt-total height of roughness profile & 6.07 & 1.08 & 0.78 \\
\hline Rp-maximum profile peak height & 1.66 & 0.27 & 0.34 \\
\hline $\mathrm{Rv}$-maximum profile valley depth & 1.07 & 0.37 & 0.28 \\
\hline Wa-arithmetic mean waviness value & 0.35 & 0.36 & 0.74 \\
\hline Wq-root mean squared waviness value & 0.41 & 0.42 & 0.86 \\
\hline $\mathrm{Wt}$-total height of the waviness profile & 1.95 & 0.95 & 4.40 \\
\hline Wp-maximum waviness peak height & 0.48 & 0.43 & 0.91 \\
\hline $\mathrm{Wv}$-maximum waviness valley depth & 0.54 & 0.44 & 1.02 \\
\hline $\mathrm{Pa}$-arithmetic mean primary profile value & 0.41 & 0.42 & 0.75 \\
\hline $\mathrm{Rq}$-root mean squared primary profile & 0.54 & 0.49 & 0.81 \\
\hline $\mathrm{Pt}$-total height of the primary profile & 6.63 & 2.55 & 4.52 \\
\hline $\mathrm{Pv}$-maximum primary profile valley depth & 1.31 & 0.76 & 1.15 \\
\hline
\end{tabular}

Figure 8a presents the gear teeth flank condition before the SF optimization. There is some visibility of the residual lines from the grinding process, as well as the waviness pattern. This surface condition creates the anisotropy of the teeth flanks. This topography is not likely. It was generating the gear teeth wear according to Figure 9. This abrasive wear phenomena appeared in the high peak of waviness areas. This kind of wear could progress and finally perform gear teeth damage. The picture of the wear (Figure 9) was performed on stereoscopic microscope with specific light. Figure 8b presents the teeth flank condition after SF process parameters optimization. There is a lack of directional waviness and residual grinding lines. The optimized surface will be tested in near future (the status on 21 February 2022) to understand the gear's performance versus non-optimized SF and the gear teeth wear development.

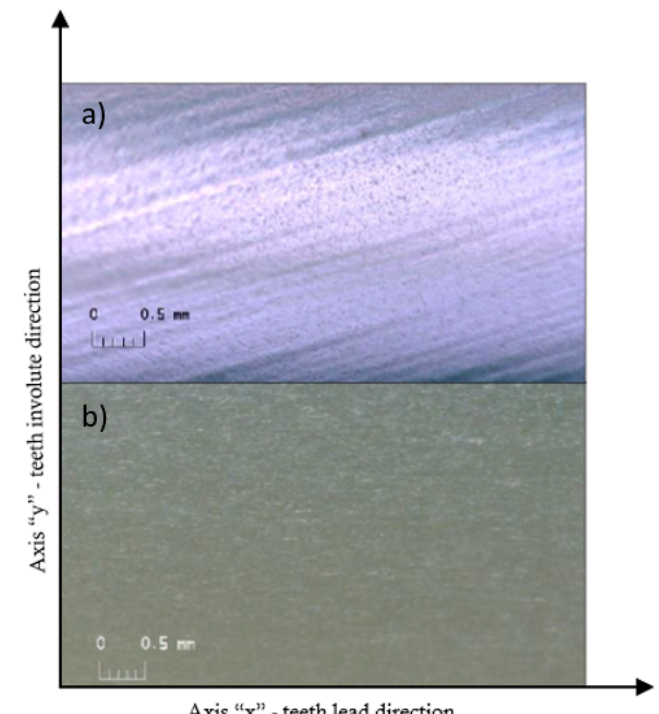

Figure 8. Gear tooth flanks visualization before (a) and after (b) abrasive process parameters optimization. 


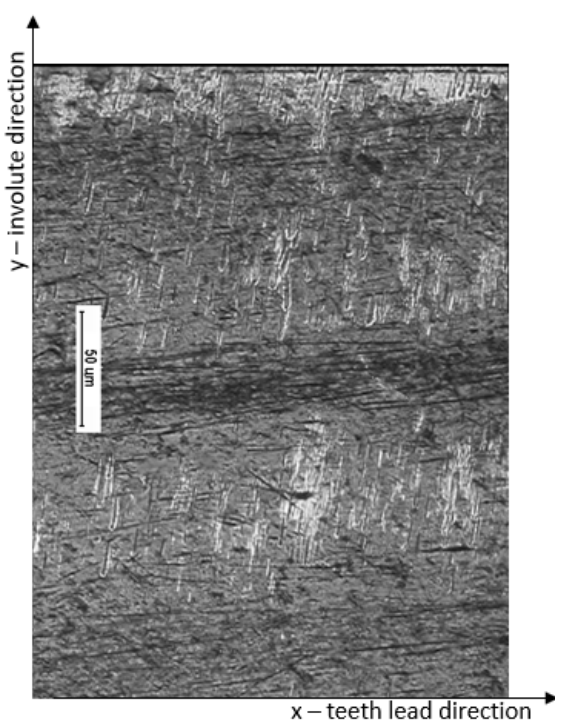

Figure 9. Non-preferable teeth flanks wear in high waviness peaks.

3D scans (confocal technics) present the initial surface condition after teeth grinding operation (Figure 10) and the difference between the non-optimized and optimized SF process in Figures 11 and 12. The condition after grinding is the non-isotropic surface topography, the peaks and valleys are parallel to each other in the grinding wheel feed direction (teeth lead) with $1 \mu \mathrm{m}$ peaks highs. The residual lines after grinding and involute waviness are visible on the surface before the SF process optimization. The examination shows that the required isotropy has not been fully achieved, a lot of scratches and waviness marks along the teeth lead are still visible. The peak highs of the irregularities are still quite large up to $0.7 \mu \mathrm{m}$. It could generate wrong contact stress distribution and risk of the gear teeth wear. The surface texture after the SF process optimization looks completely different. There is no directional pattern visibility and there is significant visibility of the surface isotropy. The irregularities were reduced to a satisfactory range (below $0.4 \mu \mathrm{m}$ ). The measured areas for the workpiece used in the confocal measurement method were rectangles of $1.5 \mathrm{~mm} \times 0.3 \mathrm{~mm}$.

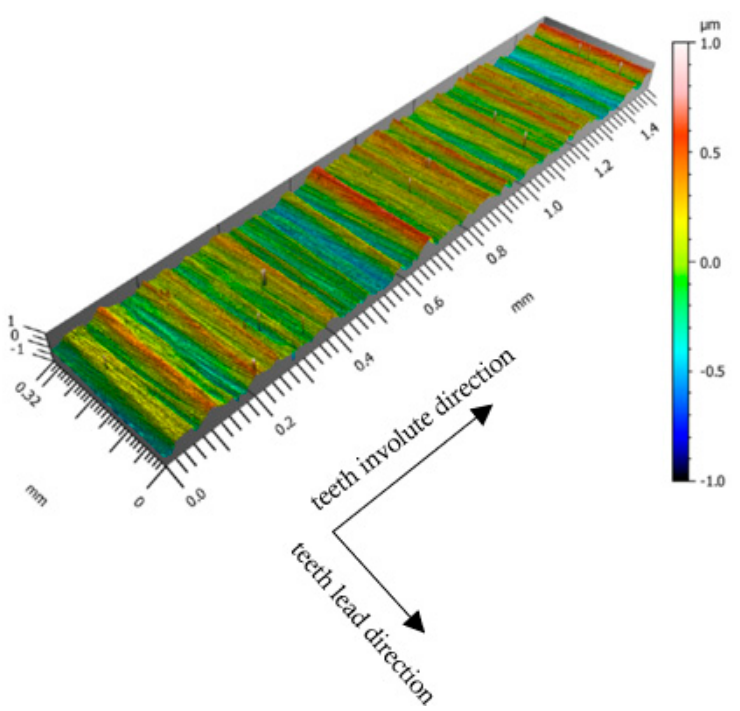

Figure 10. Visibility of the tooth flank after teeth grinding process. 


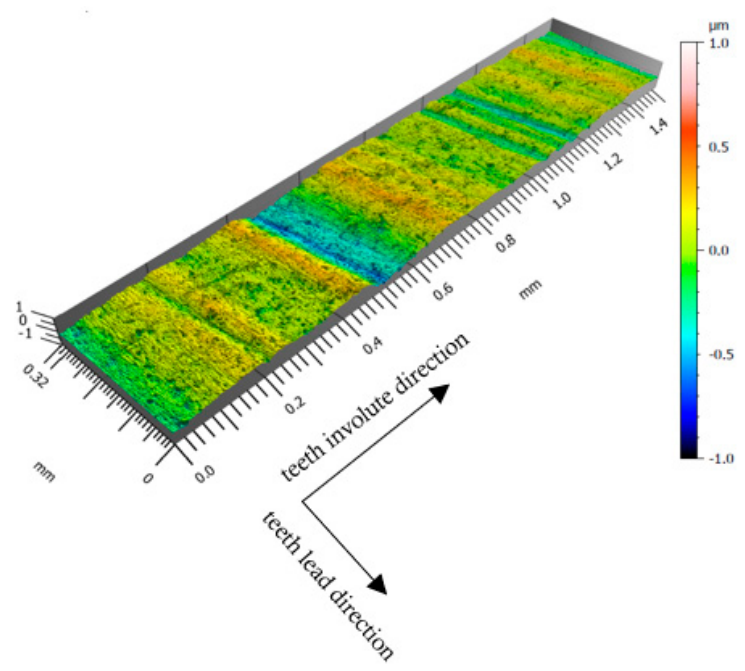

Figure 11. Visibility of the tooth flank before the abrasive process optimization (Stream Finishing).

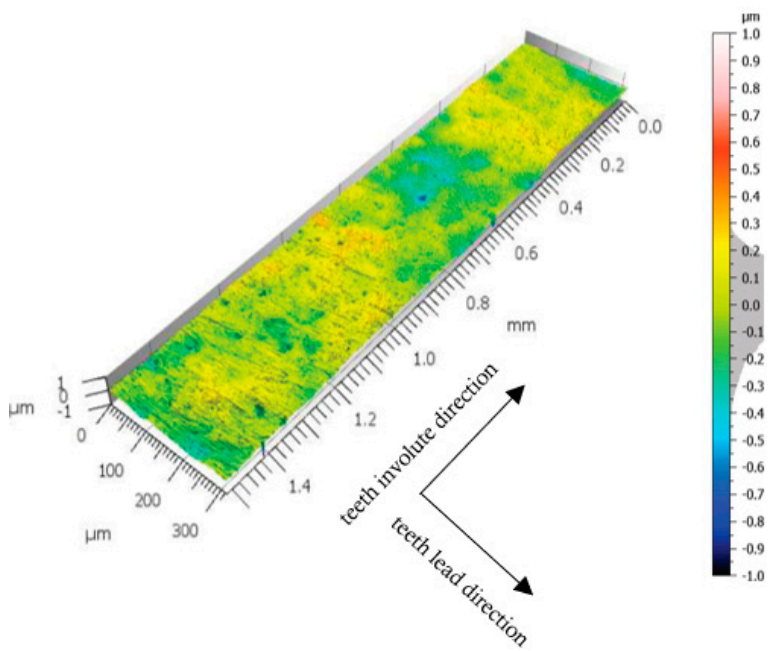

Figure 12. Visibility of the tooth flank after the abrasive process optimization (Stream Finishing).

Figure 13 presents the specific parameters on the charts which are the isotropy percentages. Before the SF process optimization that parameter is only about $8 \%$. This means that there is lack of isotropic on the gear tooth flanks. After the process optimization this parameter increased significantly up to $62 \%$.

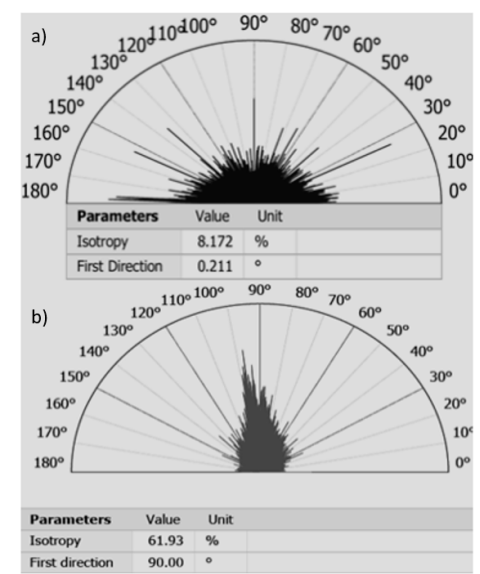

Figure 13. Isotropy chart before (a) and after (b) Stream Finishing process parameters optimization. 
In addition, very good surface visualization was made on a Talysurf CCI device in 2D. Before determination of the proper SF parameters, the Talysurf CCI presents linear, parallel shapes of the gear teeth flank topography (Figure 14a). The marks are residual from gear teeth grinding process. For the proper SF parameters, the isotropic was created and no directional topography is visible (Figure $14 \mathrm{~b}$ ). The measured areas are $2.5 \mathrm{~mm} \times 2.5 \mathrm{~mm}$ taken from pitch diameters of the workpieces. The total tooth height of the workpiece was about $7 \mathrm{~mm}$, so there was opportunity to examine almost $30 \%$ of the tooth height on this device in the involute direction. This is a quite big area of the inspection.

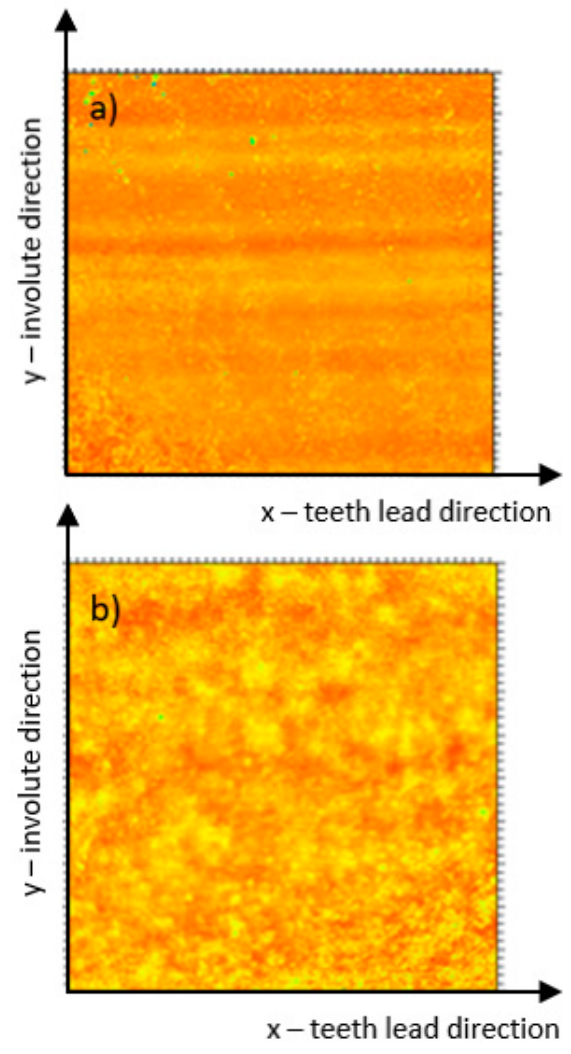

Figure 14. Isotropic surface visualization before (a) and after (b) Stream Finishing process optimization.

The medium flow direction is also the key process parameter which creates the proper surface texture. The recommended flow shall be across the directional lines to help in isotropy surface achievement. Before the SF process optimization, the medium created parallel marks in Figure 15a, but the proper process parameters determinations changed the medium flow to the recommended texture in Figure 15b. In addition, the scratches and irregularities performed by the small particles are much more significant for the optimized process. It means that the medium flow is more effective at removing the material from the gear tooth flanks rather than performing the tooth surfaces polishing (like the mirror). The Figure 15 images were made using a Scanning Electron Microscope (SEM). Axis $x$ presents $100 \mu \mathrm{m}$ measured distance along the teeth lead direction.

For the unaided eye, the irregularities on the gear teeth flanks done by non-optimized SF process parameters could also be observed. Some waviness was visible on teeth flanks when light was used at a proper angle (Figure 16a). The gear teeth flank surface after the optimized SF process does not have unexpected irregularities and there are no residual grinding process lines visible (Figure 16b). However, unaided eye inspection is not enough to properly examine the surface topography within a high-quality specification, so additional inspection techniques would be needed and defined as engineering requirements. 


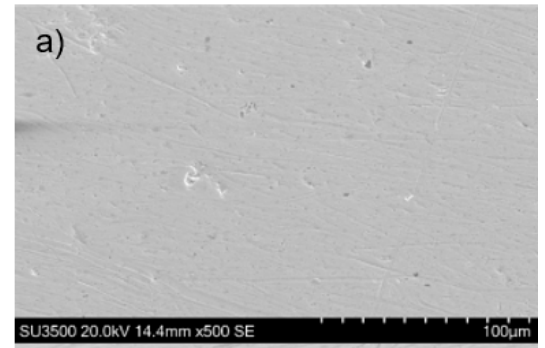

b)

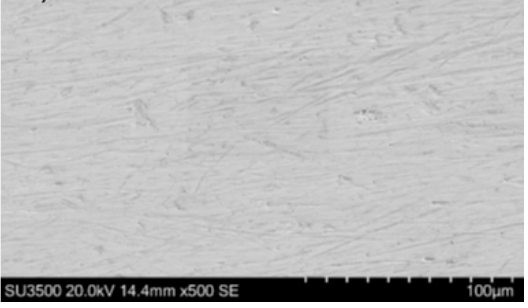

Figure 15. SEM picture before (a) and after (b) Stream Finishing process optimization.

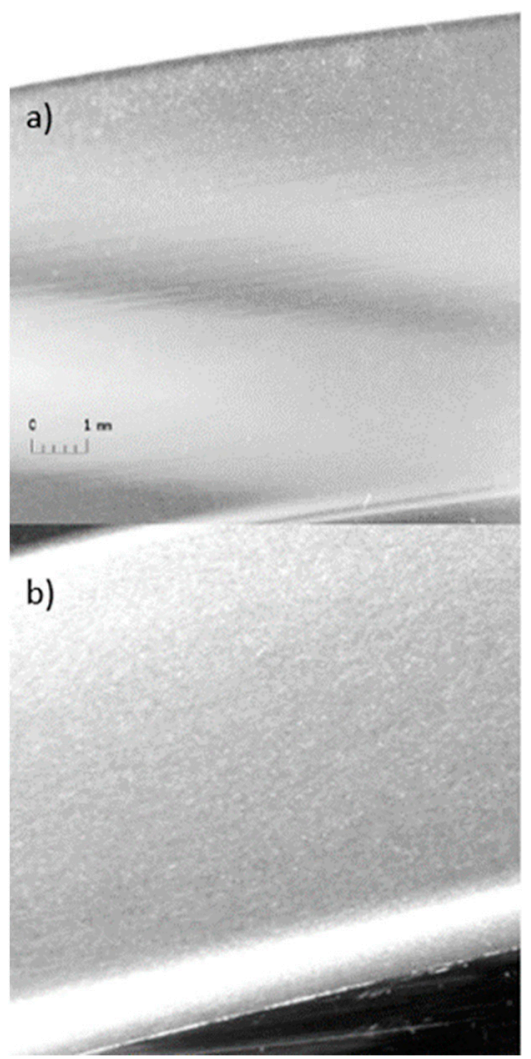

Figure 16. Unaided eye inspection for workpiece before (a) and after (b) Stream Finishing process optimization.

The hardness of the teeth flanks is very important to meet the required lifetime of the gearbox. It prevents against teeth wear. To obtain the proper high hardness of the gear teeth there is a need to use carburization process and hardening. After these operations the next step is gear teeth final grinding $[29,30]$. All distortions from the heat treatment process are removed in the grinding process. Some of the case depth variation around the gear might be visible when the same allowances are used to machine the part. The SF process also influences the case depth dimension as well as the hardness. Based on understanding the material removal rate of the optimized SF process (Figure 17), the microhardness inspection 
was done, along with a case depth inspection, to verify if minimum case depth was still met. The idea of this examination comes up with a very effective material removal rate from the gear teeth involute, especially from the tip diameter area (Figure 17). The curve in blue is the involute shape after the gear teeth grinding process. This shape is changed when SF is applied. Non-optimized SF parameters keep the involute shape within drawing tolerance due to the small influence of the medium (yellow line). For the very effective SF process (optimized parameters) there is more material removed from the gear tooth flanks. In this case the involute shape is out of the tolerance on tip diameter. This situation requires the change of the grinding wheel design. Additionally, optimized SF process also prepares a very good shape of the gear teeth top lands (very smooth and continuous shapes) without any sharp edges at the tip diameter which would risk micropitting or scuffing (Figure 18).

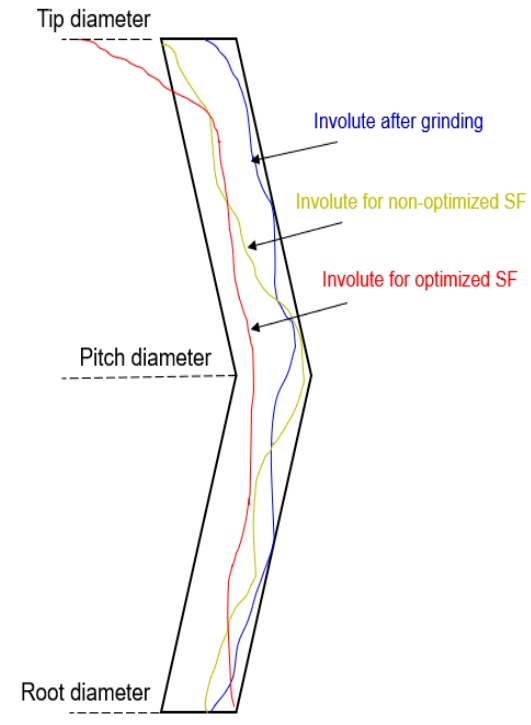

Figure 17. Involute shapes for gears after Stream Finishing process based on typical tolerances from aviation business.

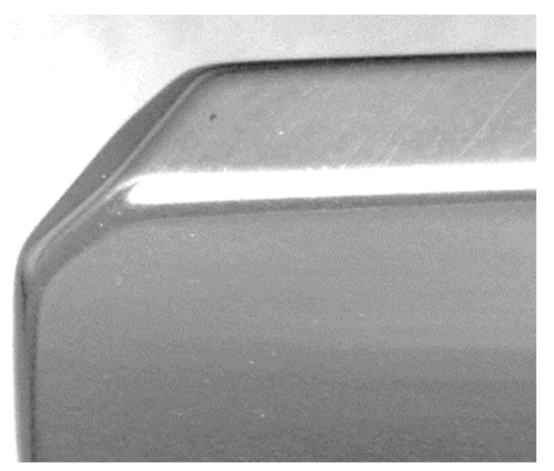

Figure 18. Perfect shapes of top lands, gear edges and chamfers areas achieved in Stream Finishing process for workpiece.

Figure 19 presents the sample of the gear which was cut to inspect the carbon distribution around the teeth. There are 1-9 localizations critical to measuring the hardness and case depth-including root diameter, root fillet radius, pitch diameter and tip diameter- to check if the optimized SF process parameters keep the required carbon depth defined on manufacturing drawing. After the analysis there is still visibility of the carbon at the tip diameter area, as well as the required hardness. 


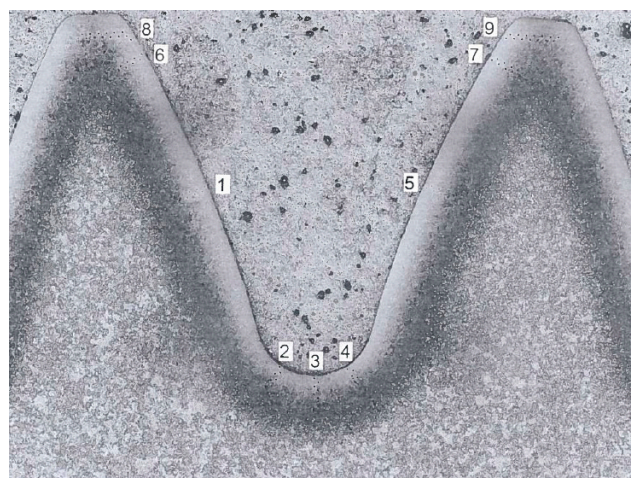

Figure 19. Carbon diffusion around the gear teeth for workpiece after the optimized Stream Finishing process.

The lubrication method of oil delivery to gear mesh is important for achieving the required lifetime of the gearbox and working without any problems in operation conditions. The surface conditions should be without any irregularities, but from the lubrication point of view there is a need to have some valleys to keep the lubricant (oil pockets) [31]. The color of the teeth flank after the SF process is metallic with reflection. To see if there is the possibility of accumulating the oil in the surface irregularities, AFM (atomic force microscope) inspection was done according to Figure 20. It was very hard to observe the oil pockets by other measurement methods.

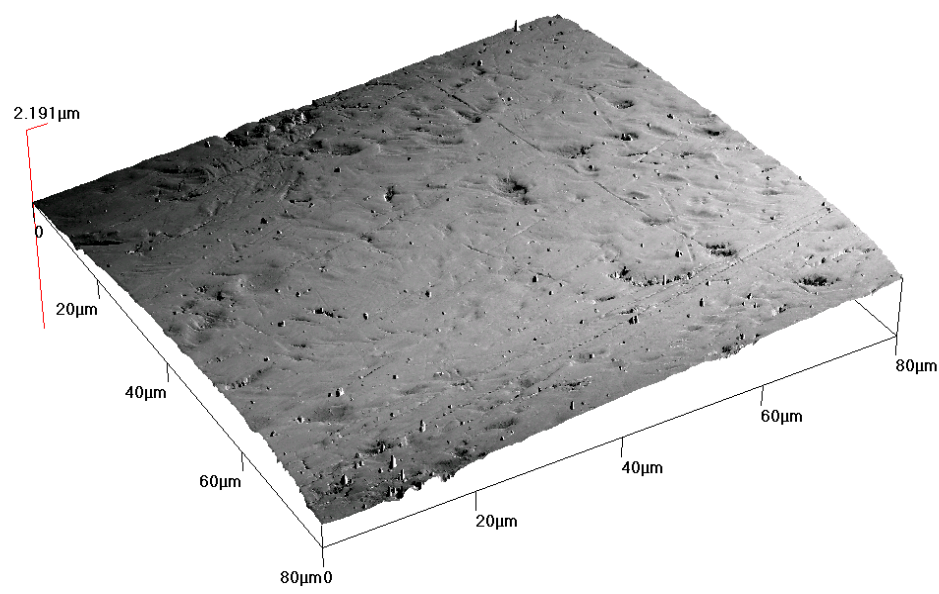

Figure 20. AFM inspection to observe the surface irregularities.

The involute process variation in gear teeth finishing methods is a key factor to determining the tolerances of the final teeth grinding process. The perfect case would be when the finishing process variation is small enough to allow grinding wheel design preparation in a wide enough tolerance range. The SF process is stable within $30 \%$ of involute tolerance for the workpiece chosen from aviation business. Figure 21 presents the SF process variation versus the involute tolerance zone defined on gear manufacturing drawing. This chart presents the visualization of the material removal rate variation along the involute. 


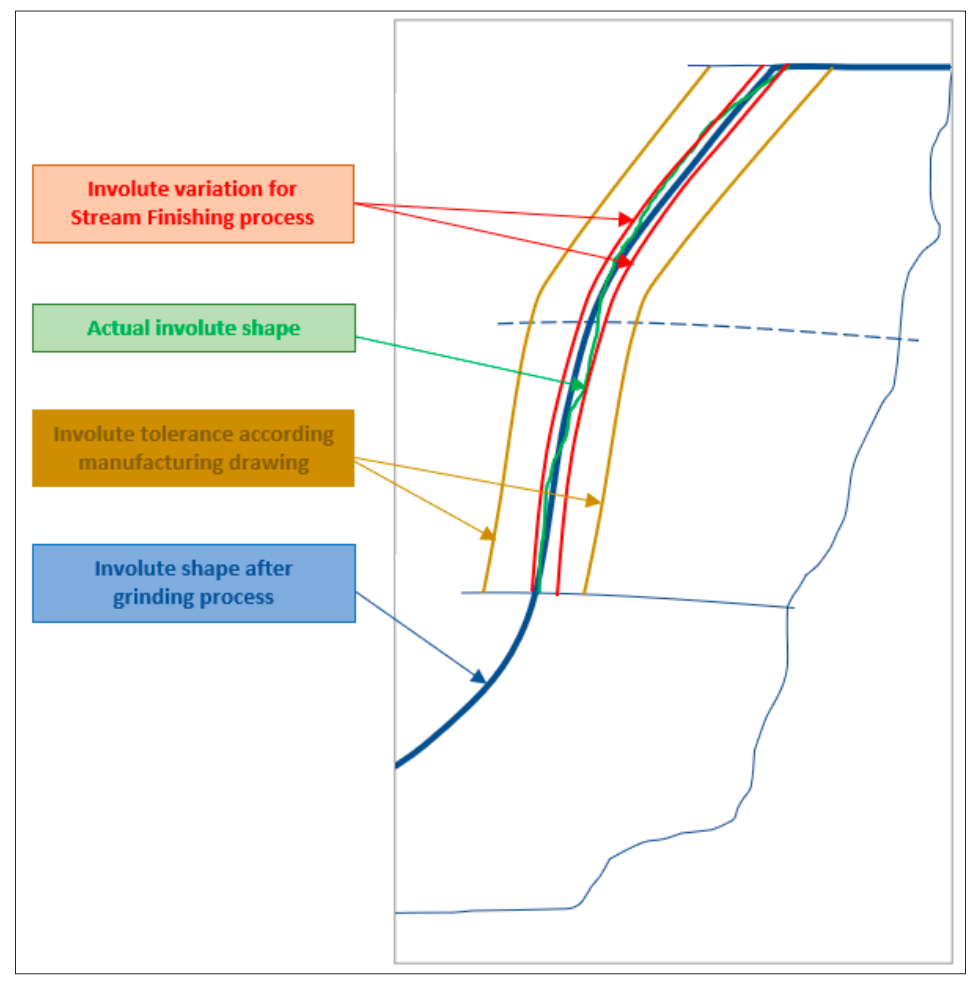

Figure 21. Involute shape variation for the Stream Finishing process.

\section{Discussion}

The Stream Finishing (SF) process described in this paper could be the additional method of gear tooth finishing applied to highly loaded gears, alongside shaving, honing, and superfinishing in a properly optimized gear manufacturing market. The results of the gear teeth flanks condition are very accurate from N5 (after grinding operation) to N3 after the SF process (ISO 1302). Furthermore, the isotropic teeth flank condition was obtained, involute waviness was reduced, and non-directional process media flow was achieved. The simplicity of the process described in this paper, as well as the easy process parameter control, are key to applying it to the manufacturing process. The environmentally friendly application, which reduces waste, is also an important factor. The small size of the media not only machines the tooth flanks to obtain the correct surface texture but also achieves the proper shape of the teeth's sharp edges. The SF process parameters should be determined individually and tested for each part number, because the wrong adjustments might give unacceptable results and negatively influence the lifetime of the gearbox.

Author Contributions: Conceptualization, W.B. and A.M.; methodology, W.B.; software, W.B.; validation, W.B. and A.M.; formal analysis, A.M.; investigation, W.B.; resources, W.B.; data curation, W.B.; writing-original draft preparation, W.B.; writing-review and editing, W.B.; visualization, W.B.; supervision, A.M.; project administration, A.M.; funding acquisition, A.M. All authors have read and agreed to the published version of the manuscript.

Funding: This research received no specific grant from any funding agency in the public, commercial, or not-for-profit sectors.

Institutional Review Board Statement: Not applicable.

Informed Consent Statement: Not applicable.

Data Availability Statement: Data is contained within the article.

Conflicts of Interest: The authors declared no potential conflict of interest with respect to the research, authorship, and/or publication of this article. 


\section{References}

1. Radzevich, S.P. Practical Gear Design and Manufacture; CRC Press: Boca Raton, FL, USA, $2012 ;$ ISBN 9781439866016.

2. Bukashkin, A.Y.; Dobretsov, R.Y.; Galyshev, Y.V. Split Transmission of Tractor with Automatic Gearbox. Procedia Eng. 2017, 206, 1728-1734. [CrossRef]

3. How Gears Work. Available online: www.howstuffworks.com (accessed on 1 October 2021).

4. Houser, D.R. The effect of manufacturing microgeometry variations on the load distribution factor and on gear contact and root stresses. Gear Technol. 2009, 6, 51-60.

5. Dav Trocoide. Android Apps for Mechanical Designers, Gear Profile Tolerances, December 2015. Available online: http: //trocoide.blogspot.com/2015/12/gear-profile-tolerances.html (accessed on 1 October 2021).

6. Morales-Espejel, G.E.; Wemekamp, A.W.; Félix-Quiñonez, A. Micro-geometry effects on the sliding friction transition in elastohydrodynamic lubrication. Proc. Inst. Mech. Eng. Part J J. Eng. Tribol. 2010, 224, 621-637. [CrossRef]

7. Lainé, E.; Olver, A.V.; Beveridge, T.A. Effect of lubricants on micropitting and wear. Tribol. Int. 2008, 41, 1049-1055. [CrossRef]

8. Rexnord Industries, LLC, Gear Group. Failure Analysis Gears-Shafts-Bearings-Seals, Failure Analysis Installation \& Maintainance, August 1978. Available online: https://www.rexnord.com/contentitems/techlibrary/documents/108-010_manual (accessed on 1 October 2021).

9. Budny, R. Five Common Gearbox Failures and How to Identify Them; North America Windpower: Honolulu, HI, USA, 2014.

10. Kim, W.; Lee, J.Y.; Chung, J. Dynamic analysis for a planetary gear with time varying pressure angles and contact ratios. J. Sound Vib. 2012, 331, 883-901. [CrossRef]

11. Lin, J.; Parker, R.G. Planetary gear parametric instability is caused by mesh stiffness variation. J. Sound Vib. 2002, 249, 129-145. [CrossRef]

12. Grzesik, W. Wpływ topografii powierzchni na właściwości eksploatacyjne części maszyn. Mechanik 2015, 88, 587-593. [CrossRef]

13. Precision Finishing. Available online: https://otecusa.com/finishing-processes/precision-finishing (accessed on 2 October 2021).

14. Bergs, T.; Klocke, F.; Brecher, C.; Schrank, M.; Kampka, M.; Kiesewetter-Marko, C.; Löpenhaus, C.; Epple, A. Predict. Process Forces Gear Honing. Gear Technol. 2019, 36, 34-39.

15. Schell, J.; Hoiland, B.; Wilmot, D.; Rindt, J.; Mitton, D.B.; Forks, G. The Fundamentals of Surface Engineering by Superfinishing using a Chemically Assisted Vibratory System. Plat. Surf. Finish. 2008, 95, 8.

16. Precision Finishing. Available online: https:/ / otecusa.com/?s=stream (accessed on 2 October 2021).

17. Precision Finishing. The Picture Was Shared by OTEC Precision Finish, Inc. for Publishing Needs; C2017 OTEC Precision Finish, Inc. I Website Built by RI Web Gurus; OTEC Precision Finish, Inc.: North Kingstown, RI, USA, 2017.

18. National Physical Laboratory. Surface Texture Measurements of Gear Surfaces Using Stylus Instruments; Good Practice Guide No. 147; Department for Business, Energy \& Industrial Strategy: London, UK, 2017.

19. Berdan, R. The Value of a Stereo Microscope, Motic America, June 2020. Available online: https://moticmicroscopes.com/blogs/ articles/the-value-of-a-stereo-microscope (accessed on 2 October 2021).

20. Elliott, A.D. Confocal Microscopy: Principles and Modern Practices. Available online: https://www.ncbi.nlm.nih.gov/pmc/ articles/PMC6961134 (accessed on 2 October 2021).

21. Inkson, B.J. 2-Scanning Electron Microscopy (SEM) and Transmission Electron Microscopy (TEM) for Materials Characterization. In Materials Characterization Using Nondestructive Evaluation (NDE) Methods; Elsevier: Amsterdam, The Netherlands, 2016.

22. Venkata, B.; Kumar, M. Friction and Wear of Materials: Principles and Case Studies. Lecture-07, Wear Mechanisms: Abrasive Wear. Available online: http:/ / www.nitttrc.edu.in/nptel/courses/video/113108083/lec7.pdf (accessed on 2 October 2021).

23. Jalili, N.; Laxminarayana, K. A review of Atomic Force Microscopy Imaging Systems: Application to Molecular Metrology and Biological Sciences, ScienceDirect, Mechatronics, October 2004. Available online: https: / /www.sciencedirect.com/science/ article/abs/pii/S0957415804000455 (accessed on 2 October 2021).

24. Accretech Tokyo Seimitsu. Explanation of Surface Characteristics Standards, Surface Texture Contour Measuring Instruments. Available online: https://inspectionengineering.com/wp-content/uploads/2018/03/SurfaceFinishExplain.pdf (accessed on 2 October 2021).

25. Singh, P.; Pungotra, H.; Kalsi, N.S. On the characteristics of titanium alloys for the aircraft applications. Mater. Today Proc. 2017, 4, 8971-8982. [CrossRef]

26. Mouritz, A.P. Introduction to Aerospace Materials, 1st ed.; Woodhead Publishing: Suite, PA, USA, 2012.

27. Errichello, B.; Muller, J. How to Analyze Gear Failures, Machinery Lubrication, NORIA. Available online: https://www. machinerylubrication.com/Read/150/gear-failures (accessed on 2 October 2021).

28. Britton, R.D.; Elcoate, C.D.; Evans, M.P.; Evans, H.P.; Snidle, R.W. Effect of Surface Finish on Gear Tooth Friction. J. Tribol. 2000, 122, 345-360. Available online: https://asmedigitalcollection.asme.org/tribology/article/122/1/354/477295/Effect-of-SurfaceFinish-on-Gear-Tooth-Friction (accessed on 2 October 2021). [CrossRef]

29. Broglie, M.J.; Smith, D.F. Gear Hardness Technology; Dudley Technical Group: San Diego, CA, USA, 1992.

30. Suliteanu, M. Minimizing Gear Distortion During Heat Treating. Gear Technol. 1996, 13, 15-19. Available online: https: / / www.geartechnology.com/articles/0396/Minimizing_Gear_Distortion_During_Heat_Treating (accessed on 2 October 2021).

31. Wang, W.; Zhao, W.; Liu, Y.; Zhang, H.; Hua, M.; Dong, G.; Tam, H.-Y.; Chin, K.-S. A Pocket-Textured Surface for Improving the Tribological Properties of Point Contact under Starved Lubrication. Materials 2021, 14, 1789. [CrossRef] [PubMed] 